\title{
Indices for Stiffness and Singularity Evaluation for Designing 5R Parallel Manipulators
}

\author{
Rogério Sales Gonçalves ${ }^{1}$, João Carlos Mendes Carvalho ${ }^{1}$, Giuseppe Carbone ${ }^{*}, 2$ and \\ Marco Ceccarelli ${ }^{*}$ \\ ${ }^{I}$ School of Mechanical Engineering, Federal University of Uberlândia, Uberlândia, Brasil \\ ${ }^{2}$ LARM, DiMSAT, University of Cassino, Cassino, Italy
}

\begin{abstract}
Parallel manipulators have various advantages over serial robots but they have a few drawbacks mainly referring to stiffness and singularities. Close to or in singular configurations the structure can not resist an external wrench applied to the mobile platform, therefore it may have uncontrolled movements. The study of the stiffness becomes of primary importance to design multibody robotic systems in order to properly choose materials, component geometry, shape and size, and the interaction of each component with others. This paper addresses the problem of a numerical evaluation of the structure stiffness by using the Matrix Structural Analysis when considering both links and joint stiffness, and by analyzing the singularity through stiffness performance indices. In order to prove the feasibility of the methodology for design purposes, the case for $5 \mathrm{R}$ parallel manipulator is formulated by comparing several stiffness performance indices.
\end{abstract}

Keywords: Parallel robots, stiffness matrix, matrix structural analysis, singularity, stiffness performance indices.

\section{INTRODUCTION}

Parallel manipulators typically consist of a moving platform that is connected to a fixed base by several serial chains, called limbs. Features of such systems can be better in stiffness and payload capacity with respect to the serial architectures, and have high velocity and acceleration during the operation. Furthermore, errors in the joints are not cumulative which contribute for its overall accuracy. Due to their characteristics they have been studied extensively both from theoretical and practical viewpoints. Prototypes have been conceived and built together with the development of theoretical investigations on kinematics and dynamics. The attention is focused on a number of possible applications such as manipulation $[1,2]$, packing and assembly/disassembly machines [3], motion simulation [4-7], milling machines [8], toys and sensors. However, they have some disadvantages such as small and complex workspace with internal singularities and the complexity of their direct kinematics [2, 9-11].

Therefore, one of the important limitations of parallel mechanisms is that they may lead to singular configurations in which the stiffness of the mechanism is compromised. The physical meaning of a singularity in kinematics refers to those configurations in which the number of degree of freedom (dof) of the mechanism changes instantaneously.

The concept of singularity has been extensively studied and several classification methods have been defined. Gosselin and Angeles [9] suggested a classification of singularities for parallel manipulators into three main groups. The first type of singularity occurs when the manipulator

*Address correspondence to this author at the LARM, DiMSAT, University of Cassino, Cassino, Italy; Tel: +39-0776-2993663; Fax: +39-07762993989; E-mails: carbone@unicas.it, ceccarelli@unicas.it reaches internal or external boundaries of its workspace and the output links loses one or more dof. Second type of singularity is related to those configurations in which the output link is locally movable even if all the actuated joints are locked. Third type is related to linkage parameters and occurs when both first and second type of singularities are involved. Tsai [12] classify the tree types of singularities by: inverse singularity; direct singularity and combined singularrity, respectively. Their methods are based on finding the roots of the determinant of the manipulator's Jacobian matrices. However, obtaining analytical expressions for the singularity loci for mechanisms with more than three dof is more difficult due to the complexity of the determinant.

Singularities are directly related to stiffness [13]. Stiffness can be defined as the capacity of a mechanical system to sustain loads without excessive changes of its geometry [14]. The changes produced on geometry, due to the applied forces, are known as deformations or compliant displacements.

Compliant displacements in a parallel robotic system produce negative effects on static and fatigue strength, wear resistance, efficiency (friction losses), accuracy, and dynamic stability (vibration). The growing importance of high accuracy and dynamic performance for parallel robotic systems has increased the use of high strength materials and lightweight designs, improving significant reduction of cross-sections and weight. Nevertheless, these solutions also increase structural deformations and may result in an intense resonance and self-excited vibrations at high speed [14].

In general, to design a high stiffness mechanism, many of its parts should be strong and then, heavy. However, to perform a task the robot motion must be as smooth as possible, without any sudden change on positions, velocities and accelerations and, to obtain optimum trajectories the mechanical energy of the actuator must be taken into 
account. This means that the robot parts should be light. These objectives are in conflict with each other, mainly in the applications where the robot should work at high velocities. Therefore, the study of the stiffness becomes of primary importance to design multibody robotic systems in order to properly choose materials, geometry components, shape and size, interaction of components with each other, mechanical transmission of mechanisms, actuators and the controller $[12,15,16]$.

Due to the importance of stiffness and singularities, attention has been addressed to formulate a standard "stiffness performance index" that can be computationally efficient, giving a direct engineering insight of the design parameter influence, and that can be translated into experimental determinations for validating a design process.

A stiffness performance index can be directly related to the stiffness matrix by means of different mathematical operators that can be applied to a matrix. Feasible choices can be the determinant, trace, norm and condition number at a given posture [12, 13, 15, 17, 18].

Similar to or in singular configurations, a parallel manipulator becomes uncontrollable. In these configurations, the mechanism tends to lose its stiffness while gaining extra degrees of freedom. Physically, when the mechanism is in a singular configuration, the structure cannot resist an external wrench applied to the end-effectors (mobile platform), therefore, it may collapse. Several approaches were presented in the literature to identify singularities of parallel manipulators. The usual approach is to find the determinant roots of the inverse Jacobian, but the expression of the determinant is relatively complicated and time-consuming, even with the aid of computational software. Indeed, the resulting equation is highly nonlinear.

This paper addresses the problem of a numerical evaluation of the stiffness and singularity of parallel manipulators by using stiffness performance indices that can be used for optimum design.

Firstly, the structure stiffness matrix is obtained using the matrix structural analysis $(M S A)$, where the whole structure is considered as being composed of elements: bars and joints connected by nodes. After several stiffness performances, indices are presented.

This methodology is applied for the symmetric 5R parallel manipulator as a practical case of study where the $M S A$ method enables to obtain its stiffness matrix, and the usual stiffness indices are compared to identify the singular configurations.

\section{MODELS FOR STIFFNESS ANALYSIS}

There are three main methods to derive a stiffness model of parallel manipulators [19]. These methods are based on the Jacobian matrix [20-22]; the Finite Element Analysis (FEA) [23-24] and the Matrix Structural Analysis (MSA) [19, 25-28].

The methods based on the Jacobian matrix are usual and are useful for the first estimation of the stiffness matrix. The uses of Finite Element Analysis models are reliable, but these models need to be remeshed over again by involving very tedious and time-consuming routines. However, these methods are well adapted to validate analytical models, or some experimental results. Methods based on matrix structural analysis are simple and easy for computational implementation.

In this paper, the stiffness matrix is obtained from the Matrix Structural Analysis (MSA), which is also known as displacement method or direct stiffness method (DSM). The methods of structural analysis are based on the idea of breaking up a complicated system into component parts, i.e., discrete structural elements, with simple elastic and dynamic properties that can be readily expressed in a matrix form. The discrete structure is composed by elements which are joined by connecting nodes. When the structure is loaded each node suffers translations and/or rotations, which depend on the configuration of the structure and boundary conditions. For example, in a fixed linkage no displacement occurs. The nodal displacement can be found from a complete analysis of the structure. The matrices representing a beam and a joint are considered as building blocks which, when fitted together in accordance with a set of rules derived from the theory of elasticity, provide the static and dynamic properties of the whole structure [25].

\subsection{Stiffness of Joint and Beam}

The stiffness of a joint is given by [28]:

$k_{j o \text { int }}=\left[\begin{array}{cc}k_{c} & -k_{c} \\ -k_{c} & k_{c}\end{array}\right]$

Where $k_{c}=\operatorname{diag}\left(k_{t x}, k_{t y}, k_{t z}, k_{r x}, k_{r y}, k_{r z}\right) ; k_{t x}, k_{t y}, k_{t z}$ are the translation stiffness and $k_{r x}, k_{r y}, k_{r z}$ are the rotational stiffness about the $x, y$ and $z$ axes.

The stiffness matrix of a three-dimensional straight bar with a uniform cross-sectional area can be expressed as

$k_{j}=\left[\begin{array}{cc}k_{b j} & -k_{b j} \\ -k_{b j} & k_{b j}\end{array}\right]$

where $k_{b j}$ is given by

$$
k_{b j}=\left[\begin{array}{cccccc}
\frac{A_{j} E_{j}}{L_{j}} & 0 & 0 & 0 & 0 & 0 \\
0 & \frac{12 E_{j} I_{z j}}{L_{j}^{3}} & 0 & 0 & 0 & \frac{6 E_{j} I_{z j}}{L_{j}^{2}} \\
0 & 0 & \frac{12 E_{j} I_{y j}}{L_{j}^{3}} & 0 & -\frac{6 E_{j} I_{y j}}{L_{j}^{2}} & 0 \\
0 & 0 & 0 & \frac{G_{j} J_{j}}{L_{j}} & 0 & 0 \\
0 & 0 & -\frac{6 E_{j} I_{y j}}{L_{j}^{2}} & 0 & \frac{4 E_{j} I_{y j}}{L_{j}} & 0 \\
0 & \frac{6 E_{j} I_{z j}}{L_{j}^{2}} & 0 & 0 & 0 & \frac{4 E_{j} I_{z j}}{L_{j}}
\end{array}\right]
$$

In Equation (3) $E_{j}$ and $G_{j}$ are the modulus of elasticity and the shear modulus of element $j$ respectively; $L_{j}$ is the beam length, $I_{y j}, I_{y z}$ are the moment of areas about $Y$ and $Z$ 
axes, respectively. $J_{j}$ is the Saint-Venant torsion constant and $A_{j}$ is the cross-sectional area.

For application of $M S A$ it is necessary to write the stiffness matrices of all elements in the same reference frame. This reference transformation must be computed for all elements before the assembly of the stiffness matrix of the structure. The transformation matrix, $T_{j}$, can be obtained from linear algebra.

Thus, the stiffness matrix of the elements in a common reference frame (elementary stiffness matrix), for segments, $k_{j}^{e}$, and for joints, $k_{j o \text { int }}^{e}$ can be expressed as

$\left[k_{j}^{e}\right]=\left[T_{j}\right]\left[k_{j}\right]\left[T_{j}\right]^{T}$

$\left[k_{\text {joint }}^{e}\right]=\left[T_{j}\right]\left[k_{\text {joint }}\right]\left[T_{j}\right]^{T}$

After obtaining the stiffness matrix of beans and joints in a common reference frame, the stiffness matrix of a structure can be obtained by using the MSA. Based on how the structure elements are connected through their nodes, it is possible to define a connectivity matrix. Since segment and joint stiffness are known, the global stiffness matrix can be obtained by a superposition procedure. The described methodology to obtain the global stiffness matrix considers the structure as free, i.e., without any motion constraints. By applying boundary conditions when the displacements are known, a new invertible stiffness matrix $K$ can be obtained, and then the compliant displacement can be computed as [28].

$\{\mathrm{U}\}=\mathrm{K}^{-1}\{\mathrm{~W}\}$

where $U$ is the compliant displacement and $W$ is the applied external wrench.

Although the FEA and MSA have the same basic equations, Eqs. (2), (3), (4) and (6), one can point out some advantages of the $M S A$ method: (a) Since a robotic structure is composed of segments and joints therefore, each segment and each joint can be modeled by only two nodes for the $M S A$ analysis. On the other hand for FEA, each beam is divided into several nodes and the joints stiffness, in general, is not considered. (b) Using a commercial FEA software (the usual procedure) one does not have the control of the solver. Whereas, in the MSA method the stiffness matrix assembly can be followed step-by-step. (c) In the FEA method, for each change of the structure configuration a remeshed must be made, increasing the computational cost. In the $M S A$ method, it is only necessary to improve the inverse kinematic model to obtain the stiffness mapping for all structure configurations.

\section{STIFFNESS PERFORMANCE INDICES}

A stiffness performance index can be directly related to the stiffness matrix by means of different mathematical operators that can be applied to a matrix. Feasible choices can be the determinant, trace, norm, and condition number at a given posture [13].

The determinant, $\operatorname{det}(K)$, of a stiffness matrix $K$ can be computed as
$\operatorname{det}(K)=\sum_{j=1}^{n} k_{i j}(-1)^{i+j} \cdot \operatorname{det} K_{-i,-j}$

where $K_{-i, j}$ represents the matrix obtained from $K$, with the removal of the $i$-th row and $j$-th column. Therefore, the determinant of $K$ can be used as a performance index to investigate synthetically the effect of the design parameters on the stiffness behavior [13].

The trace of stiffness matrix, $\operatorname{tr}(K)$, can be expressed as

$\operatorname{tr}(K)=\sum_{i, j=1}^{n} k_{i i}$

The trace can be computed as the sum of the components for compliant displacements along the principal directions. It gives a measure of the compliant displacements per unit of external wrench. Nevertheless, it is worth noting that the matrix components do not have the same dimensions, and thus, the sum has not a full physical interpretation.

A norm of a matrix is a scalar that gives some measures of the magnitude of the elements of the matrix. The norm of $K$ can also be very useful as stiffness index, since it provides a measure on how much the stiffness matrix differs from zero. In particular, the norm can be defined in various forms as outlined in the following.

The 1-norm, which represents the absolute maximum sum of columns of $K$, can be calculated as

$\|K\|_{1}=\underset{j}{\operatorname{Max}} \sum_{i=i}^{n}\left\|k_{i j}\right\|$

The Euclidean norm, also called as the 2-norm is the square root of the largest (non-negative) eigenvalue of the positive semidefinite product of the matrix by its transpose, regardless of the ordering of the factors, as:

$\|K\|_{2} \equiv \max _{i}\left\{\sqrt{\lambda_{i}}\right\}$

where symbol \|| $\|$ is the norm operator; $\left\{\sqrt{\lambda}_{i}\right\}$ is the set of non-negative eigenvalues of $K K^{T}$. It is to note that $\lambda_{\mathrm{i}}$ is identical to the square of the module of the i-th eigenvalue of $K$ itself. In this case, the norm expresses the spectral radius whose length is related to the maximum eigenvalue, that is, the value of the stiffness in the stiffest direction. A similar norm can be related to the minimum eigenvalue of the stiffness in the most compliant direction [13].

The Frobenius norm is the square root of the sum of the squares of the entries of the matrix $K$. It can be expressed in the form:

$\|K\|_{F} \equiv \sqrt{\sum_{i=1}^{n} \sum_{j=1}^{n} K_{i j}^{2}} \equiv \sqrt{\operatorname{tr}\left(K K^{T}\right)}$

The Chebyshev norm or infinity norm is the maximum absolute value of the entries of the matrix $K$ in the form:

$\|K\|_{\infty} \equiv \max _{i, j}\left\{K_{i j} \mid\right\}$

The condition number has been also proposed as a potential candidate for a local index of stiffness performance. The condition number, cond, of a square matrix is 
the product of the norm of the matrix and the norm of its inverse [29], Eq. (13).

$$
\operatorname{cond}(K)=\|K\| *\left\|K^{-1}\right\|
$$

It is worth noting that one can also define performance indices by using the Jacobian matrix in order to characterize the stiffness performance. But the analytical expression of the determinant of the Jacobian matrix is relatively complicated and time-consuming, even with the aid of symbolic computational software. In addition, the resulting equation is highly nonlinear.

Since the stiffness matrix is a function of the structure configuration, a mapping of its workspace must be obtained. Then the MSA method is applied to obtain the stiffness matrix and the stiffness performance index. The results can be used in multi-objective optimization criteria to obtain the optimum design parameters of the robot. Numerical results for a $5 \mathrm{R}$ parallel manipulator optimum design, considering the optimum workspace, stiffness and singularities are presented in [30].

\section{THE 5R SYMMETRIC PARALLEL MANIPULA- TOR}

A five-bar manipulator is a typical parallel manipulator with the minimal degrees of freedom, which can be used for positioning a point on a region of a plane. A 5R parallel manipulator consists of five bars that are connected end to end by five revolute joints, two of which are connected to the base and actuated, as shown in Fig. (1). Such a manipulator with a symmetric structure has attracted many researchers, who have investigated its position $[31,32]$, workspace [2, 33], assembly modes [33], singularities [2, 3, 27, 34-37], performance atlases [38] and kinematic design [35, 39].
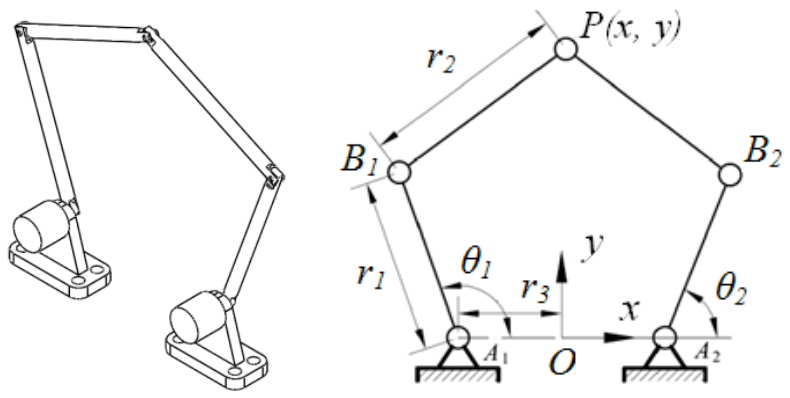

Fig. (1). The 5R parallel manipulator.

For the kinematic model, the used parameters are shown in Fig. (1). Each actuated joint is denoted as $A_{i}(i=1,2)$, the other end of each actuated link is denoted as $B_{i}$ and the common joint of the two legs is denoted as $P$, which is the output point. A fixed reference frame $O_{x y}$ is located at the center of $A_{1} A_{2}$ where the $y$ axis is normal to $A_{1} A_{2}$ and the $x$ axis is directed along $A_{1} A_{2}$. For the structure symmetry, we have $O A_{1}=O A_{2}=r_{3}, A_{1} B_{1}=A_{2} B_{2}=r_{1}$ and $B_{1} P=B_{2} P=r_{2}$. The kinematic variables are the input angles $\theta_{1}$ and $\theta_{2}$.

\subsection{Inverse Kinematic}

The input angles $\theta_{1}$ and $\theta_{2}$ can be obtained by using the inverse kinematic, when the position of point $P$ is known by considering the following restriction: $\left|p b_{i}\right|=r_{2}, \mathrm{i}=1,2$

or in the scalar form

$$
\begin{aligned}
& \left(x-r_{1} \cos \theta_{1}+r_{3}\right)^{2}+\left(y-r_{1} \sin \theta_{1}\right)^{2}=r_{2}^{2} \\
& \left(x-r_{1} \cos \theta_{2}-r_{3}\right)^{2}+\left(y-r_{1} \sin \theta_{2}\right)^{2}=r_{2}^{2}
\end{aligned}
$$

If the output point $P$ is known, the inputs angles for reaching this position can be obtained as

$\theta_{i}=2 \tan ^{-1}\left(z_{i}\right), \mathrm{i}=1,2$

where

$$
z_{i}=\frac{-b_{i} \pm \sqrt{b_{i}^{2}-4 a_{i} c_{i}}}{2 a_{i}} \quad, \mathrm{i}=1,2
$$

and

$$
\begin{aligned}
& a_{1}=r_{1}^{2}+y^{2}+\left(x+r_{3}\right)^{2}-r_{2}^{2}+2\left(x+r_{3}\right) r_{1} \\
& b_{1}=-4 y r_{1} \\
& c_{1}=r_{1}^{2}+y^{2}+\left(x+r_{3}\right)^{2}-r_{2}^{2}-2\left(x+r_{3}\right) r_{1} \\
& a_{2}=r_{1}^{2}+y^{2}+\left(x-r_{3}\right)^{2}-r_{2}^{2}+2\left(x-r_{3}\right) r_{1} \\
& b_{2}=b_{1}=-4 y r_{1} \\
& c_{2}=r_{1}^{2}+y^{2}+\left(x-r_{3}\right)^{2}-r_{2}^{2}-2\left(x-r_{3}\right) r_{1}
\end{aligned}
$$

From Eq. (18) there are four solutions for inverse kinematic problem of the $5 \mathrm{R}$ manipulator, as shown in Fig. (2a). Each solution is named as "working mode". In Fig. (2a) the working modes can be identified by $(\oplus \oplus),(\oplus \Theta),(\ominus \oplus)$ and $(\ominus \ominus)$.

\subsection{Direct Kinematic}

The direct kinematic problem consists in obtaining the coordinates of point $P$ with respect to a set of given inputs angles $\theta_{1}$ and $\theta_{2}$. From Eqs. (15) and (16) one can write

$$
\begin{aligned}
& x^{2}+y^{2}-2\left(r_{1} \cos \theta_{1}-r_{3}\right) x-2 r_{1} \sin \theta_{1} y-2 r_{1} r_{3} \cos \theta_{1}+r_{3}^{2}+r_{1}^{2}-r_{2}^{2}=0 \\
& \quad(20) \\
& x^{2}+y^{2}-2\left(r_{1} \cos \theta_{2}+r_{3}\right) x-2 r_{1} \sin \theta_{2} y+2 r_{1} r_{3} \cos \theta_{2}+r_{3}^{2}+r_{1}^{2}-r_{2}^{2}=0
\end{aligned}
$$

Equations (20) and (21) yield to

$$
x=e y+f
$$

where

$$
\begin{gathered}
e=\frac{r_{1}\left(\sin \theta_{1}-\sin \theta_{2}\right)}{2 r_{3}+r_{1} \cos \theta_{2}-r_{1} \cos \theta_{1}} \\
f=\frac{r_{1} r_{3}\left(\cos \theta_{2}+\cos \theta_{1}\right)}{2 r_{3}+r_{1} \cos \theta_{2}-r_{1} \cos \theta_{1}}
\end{gathered}
$$

The $y$ coordinate can be obtained by substituting Eq. (22) into Eq. (21) as

$d y^{2}+g y+h=0$ 
SO

$$
y=\frac{-g \pm \sqrt{g^{2}-4 d h}}{2 d}
$$

where

$$
\begin{aligned}
& d=1+e^{2} \\
& g=2\left(e f-e r_{1} \cos \theta_{1}+e r_{3}-r_{1} \sin \theta_{1}\right) \\
& h=f^{2}-2 f\left(r_{1} \cos \theta_{1}-r_{3}\right)-2 r_{1} r_{3} \cos \theta_{1}+r_{3}^{2}+r_{1}^{2}-r_{2}^{2}
\end{aligned}
$$

Equations (22) and (26) provide two solutions for the direct kinematic problem of the $5 \mathrm{R}$ manipulator. They correspond to two types of assembly modes, called the up- and down-configurations, identified by $(\oplus)$ and $(\Theta)$, respectively, as shown in Fig. (2b).

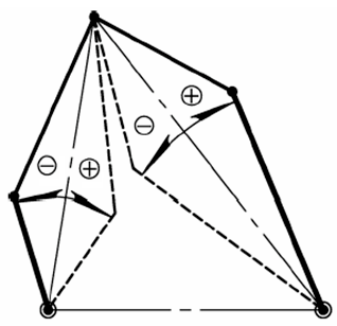

(a)

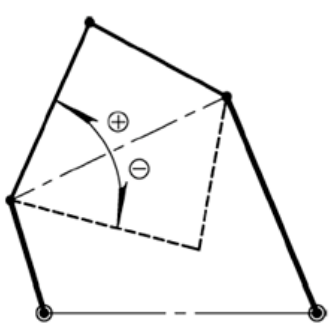

(b)
Fig. (2). (a) Working modes from inverse kinematic model; (b) Assembly modes from direct kinematic model.

\subsection{Singularity Positions of 5R Symmetric Parallel Manipulator}

In this structure the direct singularities occur when $A_{1} B_{l} P$ or $A_{2} B_{2} P$ is completely extended or folded. These singular configurations are complex configurations where the actuators cannot resist to the applied forces and/or moments on the moving platform and these loci singularities are inside the workspace. The singularities due to the inverse kinematic model correspond to the configurations in which the moving platform loses one or more degrees of freedom. These singularities occur when $A_{1} B_{1}$ is perpendicular to $B_{1} P$ or when $A_{2} B_{2}$ is perpendicular to $B_{2} P$. These singularities are on the boundary of the workspace.

In Fig. (3) the hatch region corresponds to the workspace of point $P$ to the upper assembly mode, $(\oplus)$, Fig. $(\mathbf{2 b})$, and

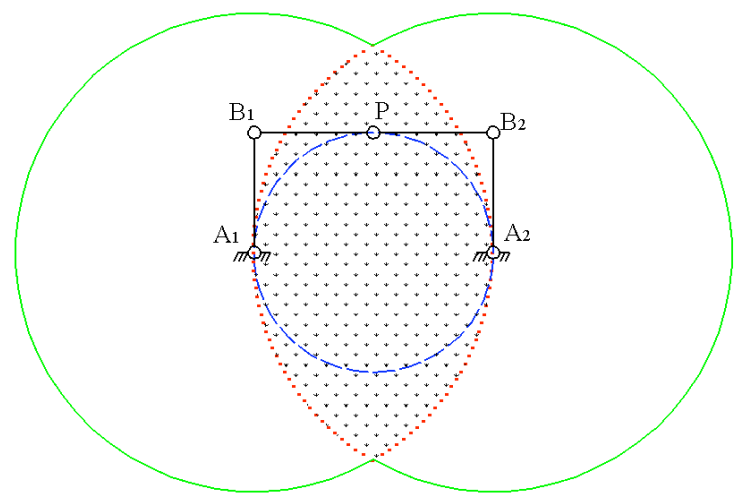

Fig. (3). Singular positions of 5R mechanism, $r_{1}=r_{2}=0,1 \mathrm{~m}$ and $r_{3}$ $=0,1 \mathrm{~m}$. the working mode, $(\ominus \oplus)$, Fig. (2a), where the boundary workspace is represented by a dotted red line. When segments $B_{1} P$ and $B_{2} P$ are aligned the singular positions are represented by a dashed blue line.

\section{MSA MODEL FOR THE 5R SYMMETRIC PARAL- LEL MANIPULATOR AND RESULTS}

Fig. (4a) shows the used nodes for the MSA model of the 5R symmetric parallel manipulator. The links $A_{1} B_{1}, B_{l} P, P B_{2}$ and $B_{2} \quad A_{2}$ are given by nodes: $1-2 ; 3-4 ; 5-6$ and 7-8 respectively. The revolute joints $B_{1}, P$ and $B_{2}$ are given by nodes: $2-3 ; 4-5$ and 6-7, respectively. The actuated joints $A_{l}$ and $A_{2}$ are given by nodes 1 and 8 , respectively. Then, the model has 8 nodes.

The segments are built with steel $\left(E=2 \times 10^{11} \mathrm{~N} / \mathrm{m}^{2}\right.$ and $\left.G=0.8 \times 10^{11} \mathrm{~N} / \mathrm{m}^{2}\right)$; the cross-sectional area is circular with $0.005 \mathrm{~m}$ diameter and $r_{1}=0.1 \mathrm{~m} ; r_{2}=0.1 \mathrm{~m}$ and $r_{3}=0.1 \mathrm{~m}$. The boundary conditions are given by actuators considered as blocked in nodes 1 and 8 . The external force and torque are applied on node 5, which is the center of the end-effector. The others joints are passive and modeled considering $k_{t x}=$ $k_{t y}=k_{t z}=2 \times 10^{11} \mathrm{~N} / \mathrm{m} ; k_{r x}=k_{r y}=2 \times 10^{11} \mathrm{~N} / \mathrm{rad}$ and $k_{r z}=$ $0 \mathrm{~N} / \mathrm{rad}$ as proposed by [28].

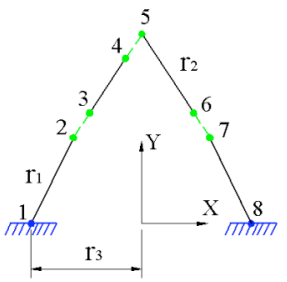

(a)

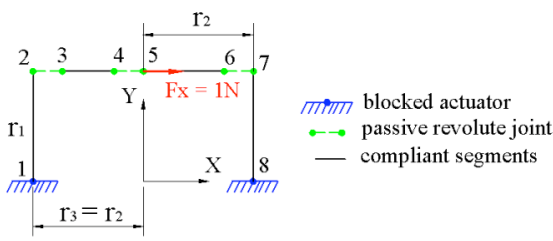

(b)
Fig. (4). (a) Nodes for the 5R mechanism; (b) 5R Mechanism in singular positions when the segments $B_{1} P$ and $P B_{2}$ are aligned.

When applying the methodology of the $M S A$ for the $5 \mathrm{R}$ manipulator, it is possible to map the stiffness using Eq. (6), simultaneously with the calculation of the stiffness performance indices, Eqs. (7) to (13).

Table 1 presented the obtained results for compliant displacements and Table 2 presented the stiffness performance indices corresponding to the same configurations. For the compliant displacements analysis, in and out of singular positions, two forces were applied on node 5, the first force in the $\mathrm{x}$-direction and the other one in the y-direction.

Whenever angles $\theta_{1}$ and $\theta_{2}$ are equal, links $B_{1} P$ and $P B_{2}$ are aligned and then, the $5 \mathrm{R}$ symmetric mechanism is in a singular position, as shown in Figs. (3 and $\mathbf{4 b}$ ) and configurations 1 to 6 in Table $\mathbf{1}$. On these configurations by applying a horizontal force, $F_{x}$, the $5 \mathrm{R}$ mechanism behaves in the same way like a 4 bar mechanism. Then, the compliant displacements, both in the $x$ and $y$ directions are insignificant.

Nevertheless, by applying a vertical force on node $5, F_{y}$, the compliant displacements in this direction are large. As the mechanism is symmetric, the compliant displacements in the $\mathrm{x}$-direction are very small.

Configurations 7 to 14 correspond to different input angles. One can see on Table 1 that the compliant displace- 
Table 1. Compliant Displacements of Node 5 for the Case of Fig. (4b) with Horizontal and Vertical Applied Forces

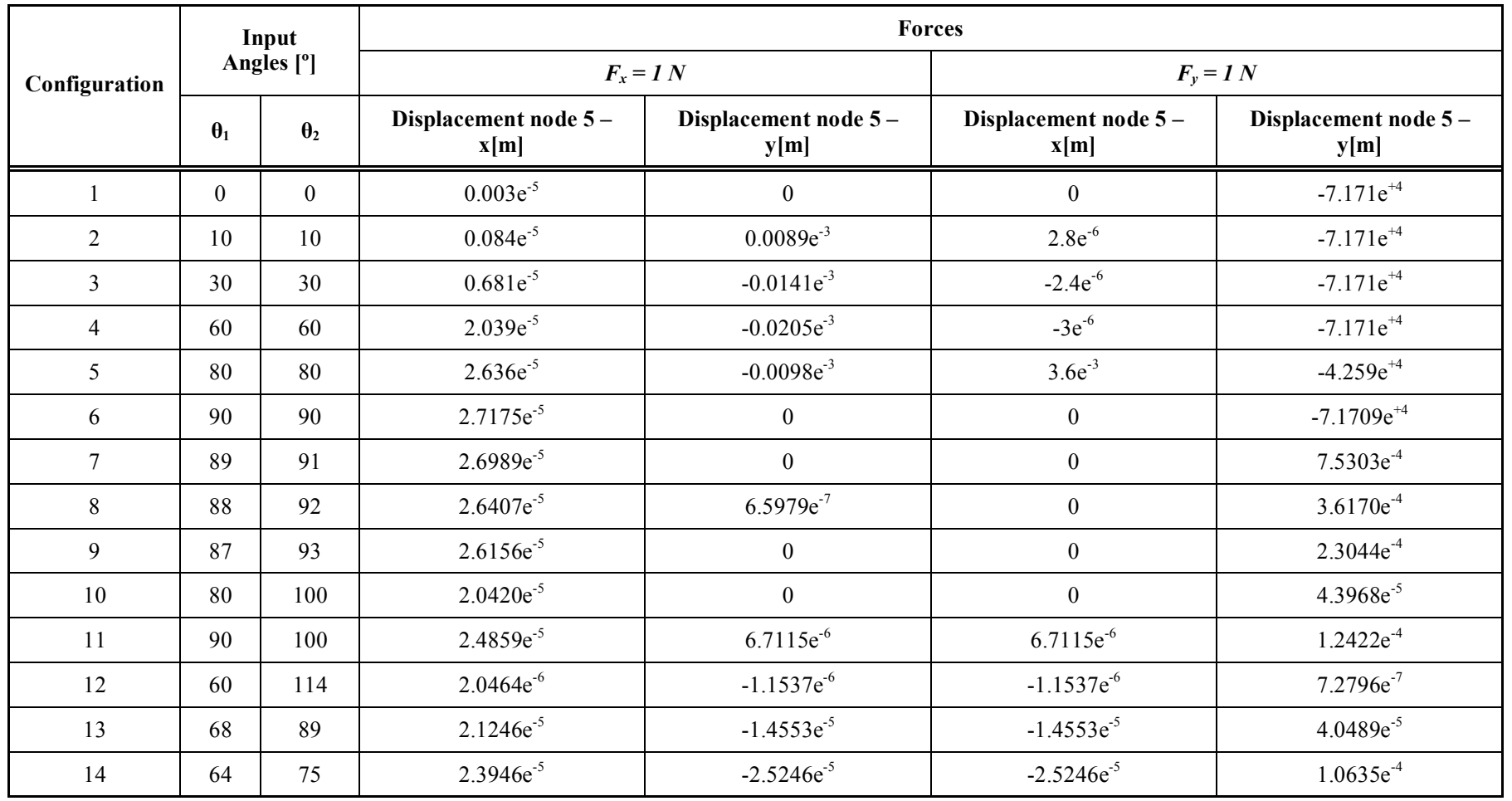

ments, both for horizontal and vertical forces, are very small. In fact these configurations do not correspond to singular positions.

Table 2 presented the values of stiffness performance indices described in section 3 , corresponding to configuretions listed on Table 1. It should be noted that the condition number gives the better index to identify the structure singularities. On these configurations the condition number is bigger than the other ones.

In a similar way the analysis can be done for singular configurations using the $M S A$ and condition number for overall structure workspace.

Table 2. Values for Stiffness Performance Indices for the 5R Parallel Manipulator of Fig. (4b) and Table 1

\begin{tabular}{|c|c|c|c|c|c|c|c|c|}
\hline \multirow{2}{*}{ Configuration } & \multicolumn{2}{|c|}{ Input Angles [ $\left.{ }^{\circ}\right]$} & \multicolumn{6}{|c|}{ Stiffness Performance Indices } \\
\hline & $\theta_{1}$ & $\theta_{2}$ & Condition Number & Determinant (K) & Trace $(\mathbf{K})$ & 2-Norm & Frobenius Norm & Chebyshev Norm \\
\hline 1 & 0 & 0 & $5.632 \mathrm{e}^{+13}$ & $-2.9785 \mathrm{e}^{+238}$ & $6.0002 \mathrm{e}^{+012}$ & $4.0007 \mathrm{e}^{+011}$ & $1.5492 \mathrm{e}^{+012}$ & $4.0008 \mathrm{e}^{+011}$ \\
\hline 2 & 10 & 10 & $6.424 \mathrm{e}^{+13}$ & $-9.9268 \mathrm{e}^{+239}$ & $6.0002 \mathrm{e}^{+012}$ & $4.0007 \mathrm{e}^{+011}$ & $1.5492 \mathrm{e}^{+012}$ & $4.0008 \mathrm{e}^{+011}$ \\
\hline 3 & 30 & 30 & $6.661 \mathrm{e}^{+13}$ & $-8.3347 \mathrm{e}^{+240}$ & $6.0002 \mathrm{e}^{+012}$ & $4.0007 \mathrm{e}^{+011}$ & $1.5492 \mathrm{e}^{+012}$ & $4.0008 \mathrm{e}^{+011}$ \\
\hline 4 & 60 & 60 & $6.661 \mathrm{e}^{+13}$ & $-2.7157 \mathrm{e}^{+241}$ & $6.0002 \mathrm{e}^{+012}$ & $4.0006 \mathrm{e}^{+011}$ & $1.5492 \mathrm{e}^{+012}$ & $4.0008 \mathrm{e}^{+011}$ \\
\hline 5 & 80 & 80 & $3.815 \mathrm{e}^{+13}$ & $-6.1253 \mathrm{e}^{+241}$ & $6.0002 \mathrm{e}^{+012}$ & $4.0006 \mathrm{e}^{+011}$ & $1.5492 \mathrm{e}^{+012}$ & $4.0008 \mathrm{e}^{+011}$ \\
\hline 6 & 90 & 90 & $5.632 \mathrm{e}^{+13}$ & $-3.7686 \mathrm{e}^{+241}$ & $6.0002 \mathrm{e}^{+012}$ & $4.0006 \mathrm{e}^{+011}$ & $1.5492 \mathrm{e}^{+012}$ & $4.0008 \mathrm{e}^{+011}$ \\
\hline 7 & 89 & 91 & $2.8782 \mathrm{e}^{+6}$ & $4.7518 \mathrm{e}^{+249}$ & $6.1374 \mathrm{e}^{+012}$ & $4.1149 \mathrm{e}^{+011}$ & $1.5847 \mathrm{e}^{+012}$ & $4.1152 \mathrm{e}^{+011}$ \\
\hline 8 & 88 & 92 & $2.9253 \mathrm{e}^{+6}$ & $1.2812 \mathrm{e}^{+250}$ & $6.2812 \mathrm{e}^{+012}$ & $4.2347 \mathrm{e}^{+011}$ & $1.6222 \mathrm{e}^{+012}$ & $4.2351 \mathrm{e}^{+011}$ \\
\hline 9 & 87 & 93 & $2.9722 \mathrm{e}^{+6}$ & $2.5953 \mathrm{e}^{+250}$ & $6.4319 \mathrm{e}^{+012}$ & $4.3603 \mathrm{e}^{+011}$ & $1.6616 \mathrm{e}^{+012}$ & $4.3607 \mathrm{e}^{+011}$ \\
\hline 10 & 80 & 100 & $3.2244 \mathrm{e}^{+6}$ & $8.0274 \mathrm{e}^{+251}$ & $7.7195 \mathrm{e}^{+012}$ & $5.4333 \mathrm{e}^{+011}$ & $2.0055 \mathrm{e}^{+012}$ & $5.4336 \mathrm{e}^{+011}$ \\
\hline 11 & 90 & 100 & $2.8058 \mathrm{e}^{+6}$ & $7.7021 \mathrm{e}^{+250}$ & $6.7417 \mathrm{e}^{+012}$ & $4.6550 \mathrm{e}^{+011}$ & $1.7433 \mathrm{e}^{+012}$ & $4.6556 \mathrm{e}^{+011}$ \\
\hline 12 & 60 & 114 & $3.4425 \mathrm{e}^{+6}$ & $2.6219 \mathrm{e}^{+255}$ & $1.4374 \mathrm{e}^{+013}$ & $1.1719 \mathrm{e}^{+012}$ & $3.8825 \mathrm{e}^{+012}$ & $1.1719 \mathrm{e}^{+012}$ \\
\hline 13 & 68 & 89 & $3.5580 \mathrm{e}^{+6}$ & $1.1066 \mathrm{e}^{+252}$ & $7.9036 \mathrm{e}^{+012}$ & $5.7669 \mathrm{e}^{+011}$ & $2.0573 \mathrm{e}^{+012}$ & $5.7674 \mathrm{e}^{+011}$ \\
\hline 14 & 64 & 75 & $3.6057 \mathrm{e}^{+6}$ & $9.3423 \mathrm{e}^{+250}$ & $6.8347 \mathrm{e}^{+012}$ & $4.7792 \mathrm{e}^{+011}$ & $1.7684 \mathrm{e}^{+012}$ & $4.7796 \mathrm{e}^{+011}$ \\
\hline
\end{tabular}


It is worth to note that the presented data on Tables $\mathbf{1}$ and 2 are obtained by numerical simulations.

\section{CONCLUSION}

This paper addresses the problem of a numerical evaluation of stiffness and singularities of parallel manipulators using stiffness performance indices.

Firstly, a methodology has been presented to obtain the stiffness matrix by using the matrix structural analysis $(M S A)$. Then, a stiffness performance index based on a condition number has been used for characterizing the stiffness and singularities of parallel manipulators both for optimum design and for operation purposes. With these results a multi-objective optimization design procedure can be formulated.

The main advantage of the $M S A$ method to obtain the stiffness matrix of a manipulator is that it is not necessary to work with differential equations, like the methods based on Jacobian matrix; the analysis of the structure does not need to remesh the system to each new position, like the Finite Element Method and, the flexibility of all elements of the structure can be considered simultaneously.

The methodology has been applied to a symmetric $5 \mathrm{R}$ parallel structure and can be applied to other structures similarly.

The reported case compares usual stiffness indices showing that using the condition number the structure singularities are efficiently identified. The other ones, i.e., $\operatorname{trace}(K), 2$ Norm, Frobenius norm and Chebyshev norm are not efficient. The determinant of the stiffness matrix can also be useful.

The mapping of the workspace of the parallel robotic structure can be elaborated, because the stiffness matrix is dependent on the structure configuration. Simultaneously, with the mapping of workspace the MSA method was applied to obtain the stiffness matrix of structure, as well as the calculation of stiffness performance index.

Thus, the MSA method associated to considerations such as workspace, stiffness and singularities can be very useful to obtain an optimum design of a parallel structure by using an adequate multi-objective optimization criteria.

\section{ACKNOWLEDGEMENTS}

The first and second authors are thankful to $\mathrm{CNPq}$, CAPES and FAPEMIG for the partial financial support of this research work. Part of this work has been elaborated within the activily of colaboration program between the Federal University of Uberlândia, Brazil, and University of Cassino, Italy.

\section{REFERENCES}

[1] R.S. Gonçalves, and J.C.M. Carvalho, "Stiffness analysis of parallel manipulator using matrix structural analysis", in EUCOMES 2008, 2-nd European Conference on Mechanism Science, Cassino, Italy, 2008, pp. 255-262.
[2] E. Macho, O. Altuzarra, C. Pinto and Hernandez A., "Workspaces associated to assembly modes of the $5 \mathrm{R}$ planar parallel manipulator", Robotica, pp. 1-9, 2008.

[3] A. Figielski, I.A. Bonev, and P. Bigras, "Towards development of a 2-DOF planar parallel robot with optimal workspace use", Systems, Man and Cybernetics, 2007.

[4] D. A Stewart, "Platform Whit Six Degrees of Freedom", Proceedings of the Institution of Mechanical Engineers, vol. 180, Pt. 1, n. 15, pp. 371-386, 1965.

[5] M. Ceccarelli, "A New 3 dof Spatial Parallel Mechanism", Mechanism and Machine Theory, vol.3 2, no. 8, pp. 895-902, 1997.

[6] N.E. Nava Rodriguez, G. Carbone and M. Ceccarelli, "CaPaMan2bis as Trunk Module in CALUMA (CAssino Low-Cost hUMAnoid Robot)", in 2nd IEEE International Conference on Robotics, Automation and Mechatronics RAM 2006, Bangkok, 2006, pp. 347-352.

[7] E. Ottaviano, and M. Ceccarelli, "An Application of a 3-DOF Parallel Manipulator for Earthquake Simulations", IEEE Transactions on Mechatronics, vol. 11, no. 2, pp. 240-146, 2006.

[8] T.A. Hess-Coelho, G.F. Batalha, D.T.B. Moraes, and M. Boczko, "A Prototype of a Contour Milling Machine Based on a Parallel Kinematic Mechanism", in Proc. of the 32nd Int. Symposium on Robotics, Seoul, Korea, 2001.

[9] C. M. Gosselin and J. Angeles, "Singularity analysis of closed loop kinematic chains," in IEEE Transactions Robot Automatic, vol. 6 , no. 3, pp. 281- 290, 1990.

[10] G. Castelli, E. Ottaviano, and M. Ceccarelli, "A Fairly General Algorithm to Evaluate Workspace Characteristics of Serial and Parallel Manipulators, International Journal Mechanics Based Design of Structures and Machines, vol. 36, pp. 14-33, 2008.

[11] R.S. Gonçalves, R.R. Santos, and J.C.M. Carvalho, "On The Performance of Strategies for the Path Planning of a 5R Symmetrical Parallel Manipulator", in DINCON 2008, $7^{\text {th }}$ Brazilian Conference on Dynamics, Control and Applications, Unesp at Presidente Prudente, SP, Brazil, 2008.

[12] L.W. Tsai, Ed., Robot Analysis: The Mechanics of Serial and Parallel Manipulators, John Wiley \& Sons, New York, 1999.

[13] G. Carbone, and M. Ceccarelli, "A comparison of indices for stiffness performance evaluation", in $12^{\text {th }}$ IFToMM World Congress, Besançon, France, paper n.A831, 2007

[14] E.I. Rivin, Ed., Stiffness and Damping in Mechanical Design, Marcel Dekker Inc., New York, 1999.

[15] M. Ceccarelli, Ed., Fundamentals of Mechanics of Robotic Manipulation, Kluwer, Dordrecht, 2004.

[16] W. K. Yoon, T. Suehiro, Y. Tsumaki and M. Uchiyama, "Stiffness Analysis and Design of a Compact Modified Delta Parallel Mechanism", Robotica, vol. 22, pp. 463-475, 2004.

[17] Y. Hou, J. Yao, L. Lu and Y. Zhao, "Performance analysis and comprehensive index optimization of a new configuration of Stewart six-component force sensor", Mechanism and Machine Theory, vol. 44, no. 2, pp. 359-368, 2009.

[18] R. Yan, F. Peng and B. Li, "General Stiffness Analysis for MultiAxis Machine Toll", in Advanced Design and Manufacture to Gain a Competitive Edge, Springer, pp. 252-261, 2008.

[19] D. Deblaise, X. Hernot, P. Maurine, "A Systematic Analytical Method for PKM Stiffness Matrix Calculation", IEEE International Conference on Robotics and Automation, 2006.

[20] D. Zhang, F. Xi, C.M. Mechefske and S.Y.T. Lang, "Analysis of parallel kinematic machine with kinetostatic modeling method", Robotics and Computer-Integrated Manufacturing, vol. 20, no. 2, pp. 151-165, 2004.

[21] F. Majou, C.M. Gosselin, P. Wenger and D. Chablat, "Parametric stiffness analysis of the orthoglide", in Proc. of the 35th International Symposium on Robotics, Paris, France, 2004.

[22] M. Ceccarelli and G. Carbone, "Numerical and experimental analysis of the stiffness performance of parallel manipulators", in 2nd International Colloquium Collaborative Research Centre 562, Braunschweig, 2005, pp.21-35.

[23] B.C. Bouzgarrou, J.C. Fauroux, G. Gogu and Y. Heerah, "Rigidity analysis of T3R1 parallel robot with uncoupled kinematics", in Proc. of the 35th International Symposium on Robotics, Paris, France, 2004.

[24] C. Corradini, J.C. Fauroux, S. Krut and O. Company, "Evaluation of a 4 degree of freedom parallel manipulator stiffness", in Proc of the $11^{\text {th }}$ World Cong. in Mechanism \& Machine Science, IFTOMM'2004, Tianjin, China, 2004 
[25] J. S. Przemieniecki, Theory of Matrix Structural Analysis, Dover Publications, Inc, New York, 1985.

[26] W. Dong, Z. Du and L. Sun, "Stiffness influence atlases of a novel flexure hinge-based parallel mechanism with large workspace", in Proc. of IEEE ICRA: Int. Conf. On Robotics and Automation, Barcelona, Spain, 2005.

[27] R. S. Gonçalves and J. C. M. Carvalho, "Singularities of Parallel Robots Using Matrix Structural Analysis", in Proceedings of the XIII International Symposium on Dynamic Problems of Mechanics - DINAME, Angra dos Reis, RJ, Brazil, 2009.

[28] R. S. Gonçalves, "Estudo de Rigidez de Cadeias Cinemáticas Fechadas", Thesis, Universidade Federal de Uberlândia, Uberlândia, Brazil (in Portuguese), 2009.

[29] C.D. Meyer, Matrix Analysis and Applied Linear Algebra, SIAM, 2000.

[30] R. S. Gonçalves and J. C. M. Carvalho, "A Multi-Objective Optimization Design for Parallel Structures", in 20th International Congress of Mechanical Engineering COBEM2009, 2009.

[31] P. M. Pardalos and Ding-Zhu Du, "Pareto Optimality, Game Theory and Equilibria", in Springer Optimization and Its Applications, Springer Science, 2008.

[32] G. Alici, "An inverse position analysis of five-bar planar parallel manipulators", Robotica, vol. 20, pp. 195-201, 2002.

[33] J.J. Cervantes-Sanchez, J.C. Hernandez-Rodriguez and J.G. Rendon-Sanchez, "On the workspace, assembly configurations and singularity curves of the RRRRR-type planar manipulator", Mechanism and Machine Theory, vol. 35, pp. 1117-1139, 2000.

[34] X.-J. Liu, J. Wang and H.-J. Zheng, "Optimum design of the 5R symmetrical parallel manipulator with a surrounded and goodcondition workspace", Robotics and Autonomous Systems, ${ }^{\circ}$ 54, pp. 221-233, 2006.

[35] J.J. Cervantes-Sanchez, J.C. Hernandez-Rodriguez and J. Angeles, "On the kinematic design of the 5R planar, symmetric manipulator", Mechanism and Machine Theory, vol. 36, pp. 13011313, 2001.

[36] T. Mbarek, G. Lonij and B. Corves, "Singularity analysis of a fully parallel manipulator with five-Degrees-of-Freedom based on Grassmann line geometry", in $12^{\text {th }}$ IFToMM World Congress, Besançon, France, 2007.

[37] S. Sun, J.W.F. Cheung, and Y. Lou, "A Study on Five-Bar Manipulators for Semiconductor Packaging Applications", in Proceedings of the 2007 IEEE International Conference on Mechatronics and Automation, China, 2007.

[38] F. Gao, X.-J. Liu and W.A. Gruver, "Performance evaluation of two-degree-of-freedom planar parallel robots", Mechanism and Machine Theory, vol. 33, no. 6, pp. 661-668, 1998.

[39] G. Alici and B. Shirinzadeh, "Optimum synthesis of planar parallel manipulators based on kinematic isotropy and force balancing", Robotica, vol. 22, pp. 97-108, 2004. 